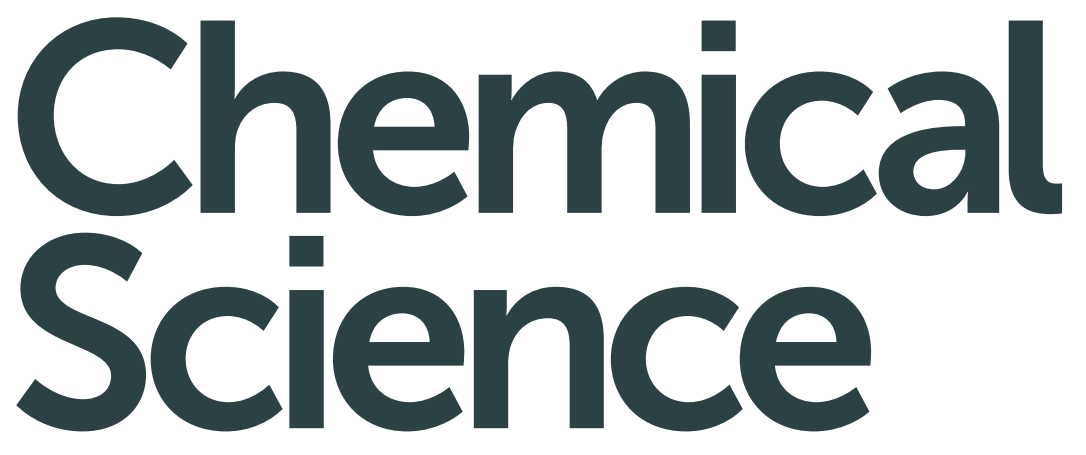

rsc.li/chemical-science

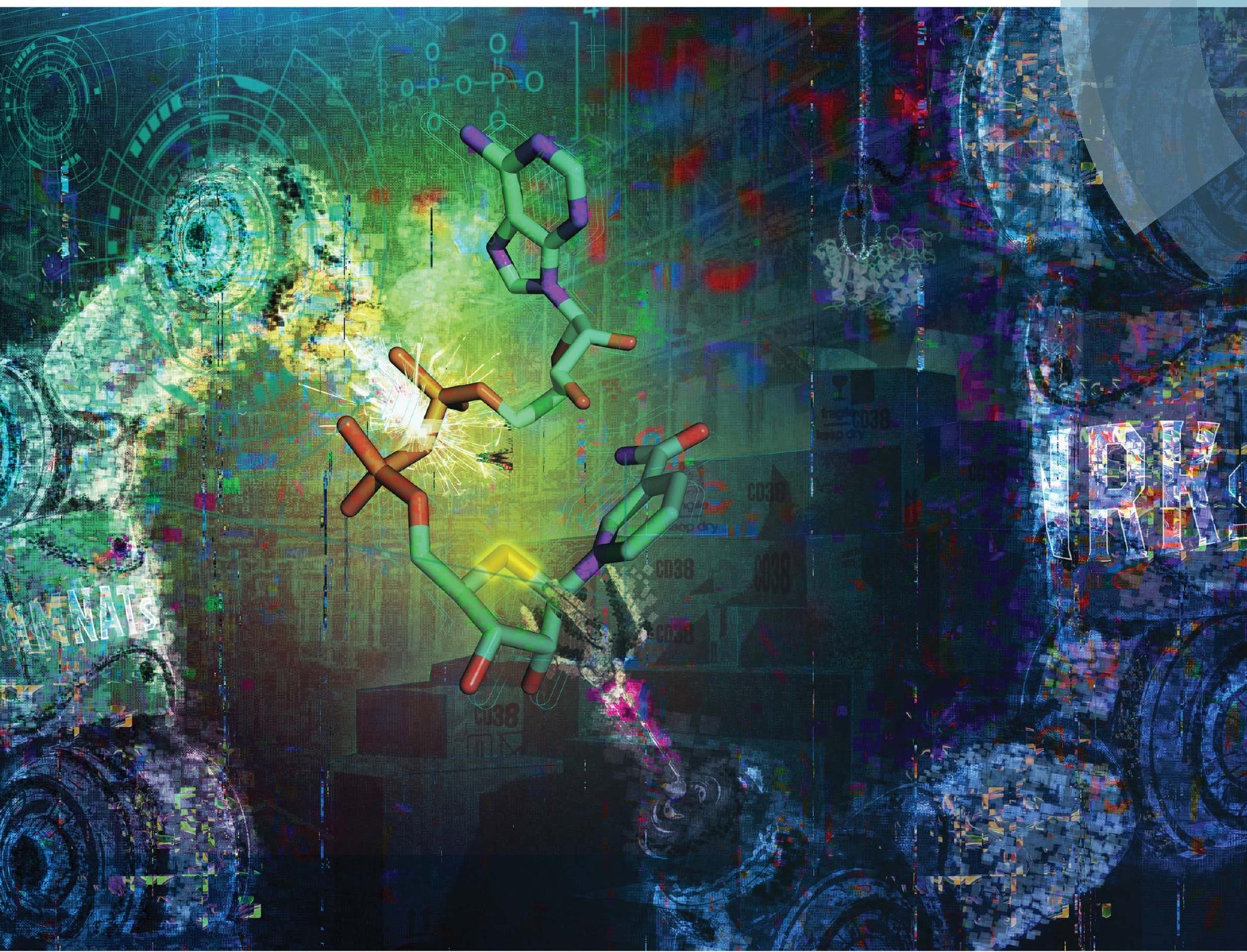

ISSN 2041-6539

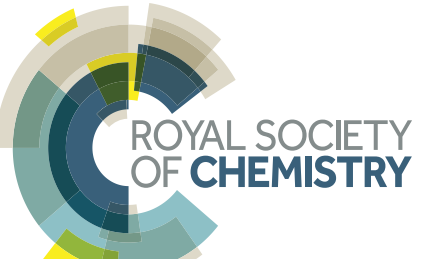


Check for updates

Cite this: Chem. Sci., 2018, 9, 8337

๑ All publication charges for this article have been paid for by the Royal Society of Chemistry

\title{
Facile chemoenzymatic synthesis of a novel stable mimic of $\mathrm{NAD}^{+}+{ }_{+}$
}

\author{
Zhefu Dai, $\S^{a}$ Xiao-Nan Zhang, $\S^{a}$ Fariborz Nasertorabi, ${ }^{b}$ Qinqin Cheng, ${ }^{a}$ Hua Pei, ${ }^{c}$ \\ Stan G. Louie, ${ }^{c}$ Raymond C. Stevens ${ }^{\star b}$ and Yong Zhang (D) *adef
}

Nicotinamide adenine dinucleotide $\left(\mathrm{NAD}^{+}\right)$is an essential cofactor participating in a variety of important enzyme-catalyzed physiological and pathophysiological processes. Analogues of $\mathrm{NAD}^{+}$provide key and valuable agents for investigating $\mathrm{NAD}^{+}$-dependent enzymes. In this study, we report the preparation of a novel stable $\mathrm{NAD}^{+}$mimic, $4^{\prime}$-thioribose $\mathrm{NAD}^{+}\left(\mathrm{S}-\mathrm{NAD}^{+}\right)$, using a facile and efficient chemoenzymatic approach. Substrate activity assays indicated the resulting S-NAD ${ }^{+}$is chemically inert to human $\mathrm{CD} 38$ and sirtuin 2 enzymes, but capable of participating in redox reactions in a manner similar to $\mathrm{NAD}^{+}$. X-ray crystallographic analysis revealed binding of S-NAD ${ }^{+}$to the active site of human CD38 and critical residues involved in leaving group activation and catalysis. By more closely mimicking $\mathrm{NAD}^{+}$in geometry and electrostatics, the generated $\mathrm{S}^{-} \mathrm{NAD}^{+}$offers a unique and important tool that can be extended to study enzymes utilizing NAD ${ }^{+}$.

Received 31st August 2018

DOI: $10.1039 / \mathrm{c} 8 \mathrm{sc} 03899 \mathrm{f}$

rsc.li/chemical-science

covalently add the ADP-ribose moiety of $\mathrm{NAD}^{+}$to side chains of target proteins, resulting in protein ADP-ribosylation. This is catalyzed by a superfamily of ADP-ribosyltransferases (ARTs), including intracellular poly-ADP-ribose polymerases (PARPs) and sirtuins and ecto-ARTs. The last category of $\mathrm{NAD}^{+}$-dependent enzymes includes CD38 and CD157 catalyzing formation of ADP-ribose (ADPR) and cyclic ADPR (cADPR) by consuming $\mathrm{NAD}^{+}$(Fig. 1). As membrane glycoproteins, CD38 and CD157 are multifunctional enzymes. In addition to catalyzing formation of cADPR from $\mathrm{NAD}^{+}$, they possess $\mathrm{NAD}^{+}$glycohydrolase and base exchange activities. Compared with CD157, CD38 exhibits significantly higher enzymatic activity and catalytic efficiency in cleaving circulating $\mathrm{NAD}^{+}$, suggesting a major role in controlling tissue and cellular $\mathrm{NAD}^{+}$homeostasis., ${ }^{\mathbf{5}, 6}$ Recent studies indicated that CD38 deficiency in mouse leads to ameliorated pathology of Alzheimer's disease. ${ }^{7}$ Pharmacological inhibitions

${ }^{a}$ Department of Pharmacology and Pharmaceutical Sciences, School of Pharmacy, University of Southern California, 1985 Zonal Ave, Los Angeles, CA 90089, USA. E-mail:yongz@usc.edu

${ }^{b}$ Departments of Biological Sciences and Chemistry, Bridge Institute, Michelson Center for Convergent Bioscience, University of Southern California, Los Angeles, CA 90089, USA.E-mail: stevens@usc.edu

'Titus Family Department of Clinical Pharmacy, School of Pharmacy, University of Southern California, 1985 Zonal Ave, Los Angeles, CA 90089, USA

${ }^{d}$ Department of Chemistry, Dornsife College of Letters, Arts and Sciences, University of Southern California, Los Angeles, CA 90089, USA

${ }^{e}$ Norris Comprehensive Cancer Center, University of Southern California, Los Angeles, CA 90089, USA

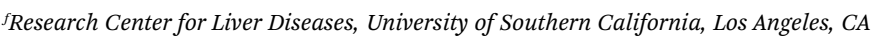
90089, USA

$\uparrow$ The X-ray structure of S-NAD ${ }^{+}$with human CD38 is deposited in Protein Data Bank (https://www.rcsb.org) (PDB ID: 6EDR).

\$ Electronic supplementary information (ESI) available: Experimental procedures, and supplementary figures, table, and data. See DOI: 10.1039/c8sc03899f

$\S$ These authors contributed equally to this work.

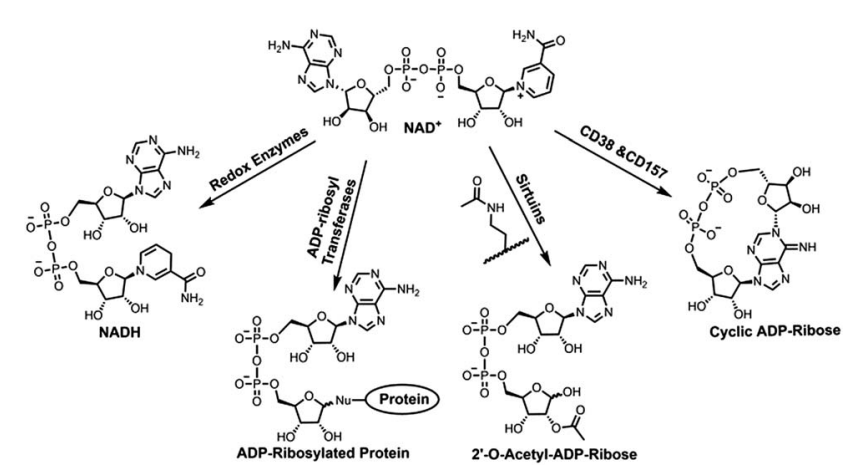

Fig. $1 \mathrm{NAD}^{+}$participates in chemical reactions catalyzed by distinct classes of enzymes. 
physiological and metabolic parameters, and provide beneficial effects on metabolic syndrome. ${ }^{8-11}$ CD38 was also shown to regulate immune responses in physiological and pathological conditions. ${ }^{12,13}$

Given the extensive involvements of $\mathrm{NAD}^{+}$-dependent enzymes in human diseases, a substantial amount of studies have been performed to determine their mechanisms of action. For this purpose, X-ray crystal structures of reactive complexes can provide direct and valuable information at the molecular levels. ${ }^{14}$ Since the complexes of $\mathrm{NAD}^{+}$-bound catalytically active enzymes are short-lived, catalytically inactive enzymes or modified substrate proteins were used for solving crystal structures of such binary or ternary enzyme complexes, which often miss important information about functions and roles of key residues involved in enzyme catalysis. ${ }^{15-17}$ Moreover, currently available $\mathrm{NAD}^{+}$analogues for this purpose are suboptimal in mimicking $\mathrm{NAD}^{+}$due to the lack of nicotinamide leaving group for redox reactions or the altered ribosyl geometry by methylene substitution. ${ }^{18-23}$ Herein we report chemoenzymatic synthesis of a novel stable $\mathrm{NAD}^{+}$mimic, $4^{\prime}$-thioribose $\mathrm{NAD}^{+}\left(\mathrm{S}^{-N A D}{ }^{+}\right)$, which was generated through two-step enzymatic reactions in a high yield following chemical synthesis of nicotinamide $4^{\prime}$-thioriboside (S-NR). Biochemical studies indicated that the resulting $\mathrm{S}^{-\mathrm{NAD}^{+}}$is resistant to enzymatic cleavage by human CD38 and sirtuin 2 enzymes, but still able to participate in redox reactions. The determined high-resolution $\mathrm{X}$-ray crystal structure of $\mathrm{S}^{-\mathrm{NAD}^{+}}$with human $\mathrm{CD} 38$ revealed molecular interactions between $\mathrm{S}^{-\mathrm{NAD}^{+}}$and key catalytic residues. These results demonstrate a facile chemoenzymatic approach for the preparation of S-NAD ${ }^{+}$. As a novel stable $\mathrm{NAD}^{+}$ mimic, the generated S-NAD ${ }^{+}$may offer a unique and important tool that can be extended to investigate mechanisms of action for enzymes utilizing $\mathrm{NAD}^{+}$.

\section{Results and discussion}

Considering the chemical structure of $\mathrm{NAD}^{+}$, we envisioned that replacing the endocyclic oxygen with sulfur can possibly generate a stable analogue more closely mimicking $\mathrm{NAD}^{+}$ in geometry and electrostatics. Furthermore, inspired by nicotinamide riboside (NR) kinase (NRK)- and nicotinamide mononucleotide adenylyltransferase (NMNAT)-mediated biosynthesis of $\mathrm{NAD}^{+}$from its NR precursor, we hypothesized that S-NR may be enzymatically converted to S-NAD ${ }^{+}$in high efficiency by human NRK and NMNAT enzymes (Fig. 2A). The human genome encodes two NRK isoforms (NRK1 and 2) and three NMNAT isoforms (NMNAT1-3). Previous studies indicated that human NRK1 and NMNAT1 exhibit adequate catalytic activities for NR+adenosine triphosphate (ATP) and nicotinamide mononucleotide (NMN)+ATP, respectively, and promiscuity towards other substrate analogues. ${ }^{24-26}$ In contrast to total chemical synthesis of $\mathrm{NAD}^{+}$analogues which has proven challenging due to synthetic complexity and low yields for the difficult pyrophosphate coupling, using recombinant NRK and NMNAT may afford a facile and efficient approach for the generation of stable $\mathrm{NAD}^{+}$mimics.
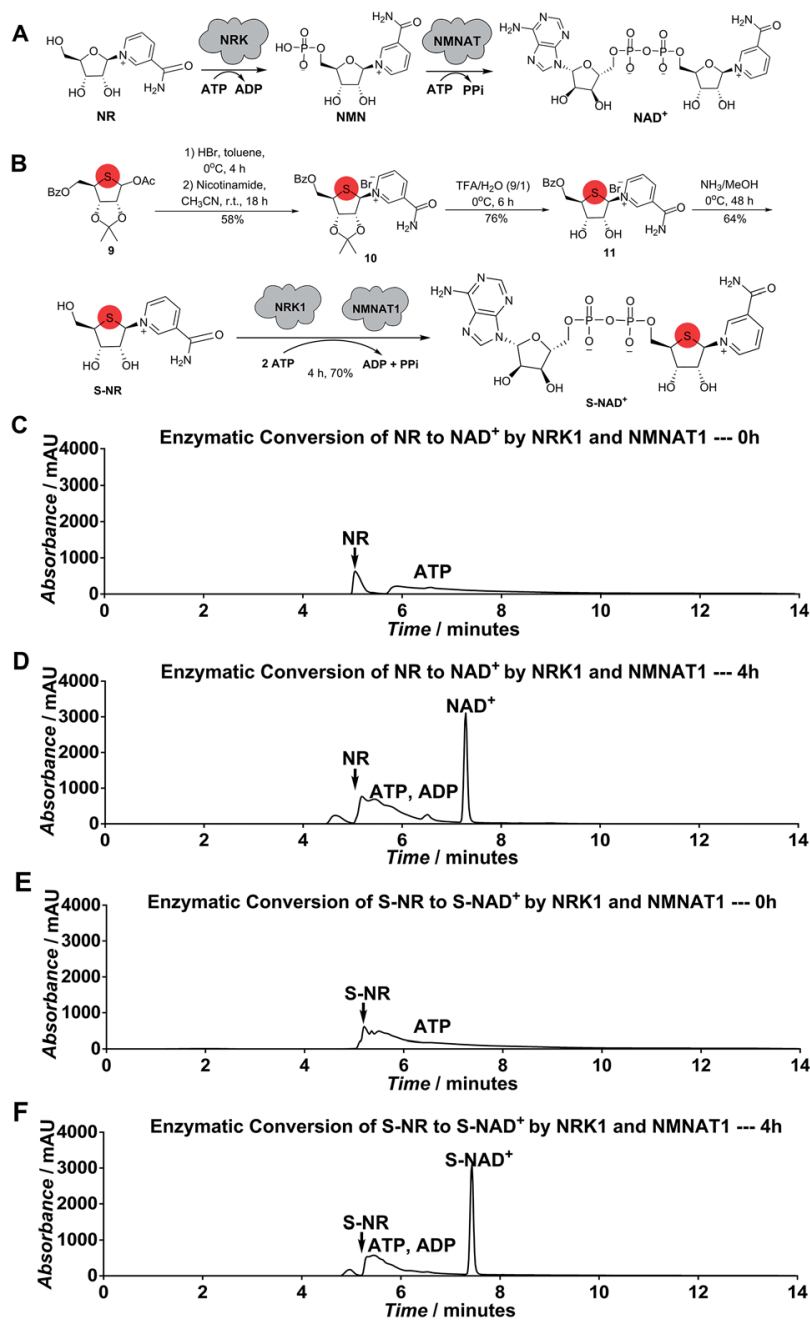

Fig. 2 Enzymatic synthesis of $\mathrm{NAD}^{+}$and chemoenzymatic synthesis of $\mathrm{S}-\mathrm{NAD}^{+}$. (A) Scheme of enzymatic conversion of $N R$ to $N A D^{+}$. (B) Scheme of chemoenzymatic synthesis of S-NAD ${ }^{+}$. (C)-(F) HPLC analysis of enzymatic synthesis of $\mathrm{NAD}^{+}((\mathrm{C})$ and $(\mathrm{D}))$ and $\mathrm{S}-\mathrm{NAD}^{+}((\mathrm{E})$ and (F)) by human NRK1 and NMNAT1 as measured by UV absorbance at $260 \mathrm{~nm} .2 \mathrm{mM}$ NR or S-NR was incubated with $6 \mathrm{mM}$ ATP and $5 \mu \mathrm{M}$ NRK1 and $5 \mu \mathrm{M}$ NMNAT1 at RT for $0 \mathrm{~h}$ and $4 \mathrm{~h}$, followed by HPLC analysis.

To test this notion, S-NR was first chemically synthesized. As shown in Scheme S1, 1-acetoxy thioribose $\mathbf{9}$ was prepared from D-gulonic acid $\gamma$-lactone according to the reported procedures. ${ }^{27}$ Despite established methods for synthesis of NR via introduction of nicotinamide onto ribose, S-NR is yet to be developed. ${ }^{28-31}$ By testing the substitution of nicotinamide for OAc group of 9, it was found that treatment of 9 with sequential $\mathrm{HBr}$ (33\% (wt) in acetic acid) in toluene at $0{ }^{\circ} \mathrm{C}$ for 4 hours and nicotinamide in $\mathrm{CH}_{3} \mathrm{CN}$ at room temperature for 18 hours successfully afforded 10 in a 58\% yield (Fig. 2B). S-NR was then generated through subsequent deprotections of the $4^{\prime}$-thioribose ring.

Human NRK1 and NMNAT1 were then expressed and purified from Escherichia coli (Fig. S1 $\$$ ). In vitro biosynthesis of $\mathrm{NAD}^{+}$ and $\mathrm{S}^{-\mathrm{NAD}}{ }^{+}$from NR and S-NR, respectively, were carried out using purified NRK1 and NMNAT1. In the presence of NRK1 
and NMNAT1 and ATP, a large amount of $\mathrm{NAD}^{+}$and S-NAD ${ }^{+}$ were rapidly formed within 4-hour incubation (Fig. 2B-F and $\mathrm{S} 2-\mathrm{S} 4+$ ). The formation rate of S-NAD ${ }^{+}$was comparable to that of $\mathrm{NAD}^{+}$. In contrast to the last two steps of chemical synthesis of $\mathrm{NAD}^{+}$analogues that usually take two to four days and are characterized by tedious purification procedures and low yields, enzymatic conversion of S-NR to S-NAD ${ }^{+}$by NRK1 and NMNAT1 could be completed within four hours with a final yield of $70 \%$ and requires significantly less efforts on purification (Fig. 2B and Scheme S1 $\$$ ). These results demonstrate a facile and efficient chemoenzymatic approach for the generation of S-NAD ${ }^{+}$. The sulfur substitution seemed to have no effect on substrate activities of S-NR and $4^{\prime}$-thioribose nicotinamide mononucleotide (S-NMN) for human NRK and NMNAT, respectively. In addition, the UV absorption spectrum of the generated $\mathrm{S}^{-\mathrm{NAD}^{+}}$is nearly identical to that of $\mathrm{NAD}^{+}$(Fig. S5 $\$$ ).

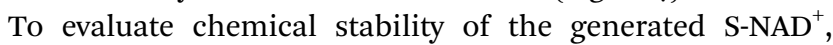
recombinant extracellular domain of human CD38 was chosen as a model enzyme (Fig. S6 $\$$ ), which catalyzes rapid formation of ADPR and CADPR from NAD ${ }^{+}$. HPLC analysis revealed that in contrast to $\mathrm{NAD}^{+}$that was completely consumed by human $\mathrm{CD} 38$ after an overnight reaction, $\mathrm{S}^{-\mathrm{NAD}^{+}}$still remained unchanged after overnight incubation with CD38 (Fig. 3). These results indicate that relative to $\mathrm{NAD}^{+}$, the synthesized $\mathrm{S}-\mathrm{NAD}^{+}$is more resistant to cleavage catalyzed by CD38 enzyme and support that 4 '-thioribose substitution for NR ribosyl group

A

A

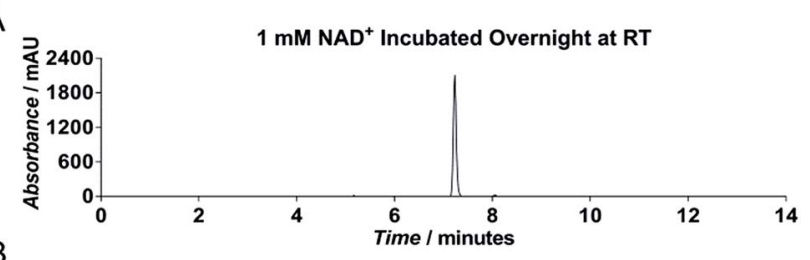

B

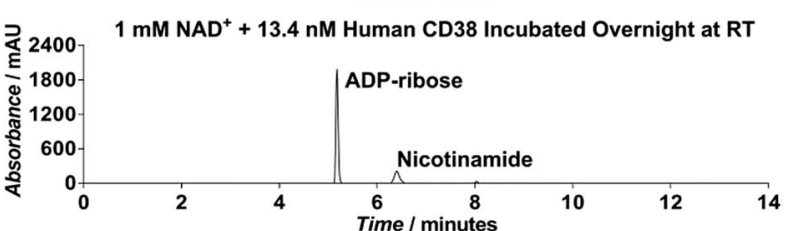

C

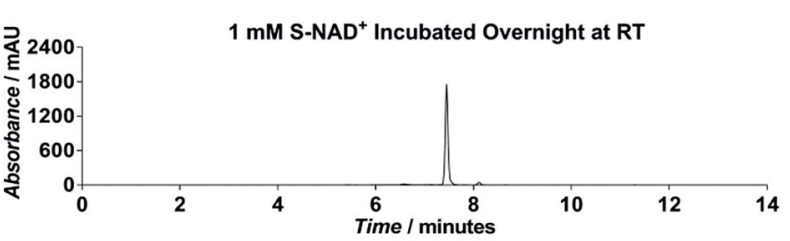

D

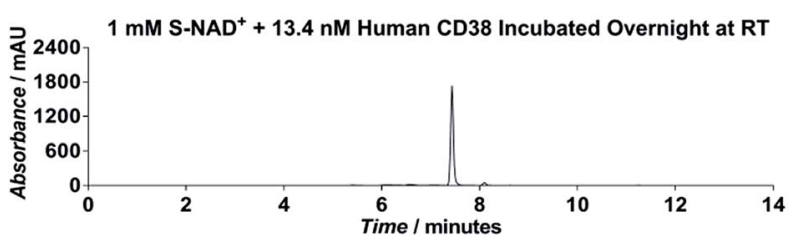

Fig. 3 Activity of $\mathrm{NAD}^{+}$and S-NAD ${ }^{+}$for human CD38. HPLC analysis of substrate activities of $\mathrm{NAD}^{+}(\mathrm{A}$ and $\mathrm{B})$ and $\mathrm{S}-\mathrm{NAD}^{+}(\mathrm{C}$ and $\mathrm{D})$ with human CD38 as measured by UV absorbance at $260 \mathrm{~nm} .1 \mathrm{mM} \mathrm{NAD^{+ }}$ or $\mathrm{S}^{-\mathrm{NAD}^{+}}$was incubated without and with $\mathrm{CD} 38$ at RT overnight, followed by HPLC analysis. results in an $\mathrm{NAD}^{+}$analogue chemically inert to catalysis for $\mathrm{N}$ glycosidic bond breakage.

Next, the generated S-NAD ${ }^{+}$was examined for its competitive inhibition activity for human CD38 enzyme by performing a continuous fluorescence-based activity assay using nicotinamide guanine dinucleotide $\left(\mathrm{NGD}^{+}\right)$as a substrate. The cyclase activity of CD38 catalyzes formation of fluorescent cyclic GDPribose (cGDPR) from $\mathrm{NGD}^{+} .{ }^{32}$ On the basis of cGDPR-derived fluorescence, kinetics for human CD38-catalyzed conversion of $\mathrm{NGD}^{+}$to cGDPR was examined and the $K_{\mathrm{m}}$ of $\mathrm{NGD}^{+}$for human CD38 cyclase activity was determined to be $8.5 \pm 0.6 \mu \mathrm{M}$ (Fig. S7 ). Competitive inhibition assays showed that S-NAD ${ }^{+}$ exhibits a dose-dependent inhibition of cGDPR formation by CD38 with a $K_{\mathrm{i}}$ of $28.5 \pm 3.1 \mu \mathrm{M}$ (Fig. 4), comparable to previously determined $K_{\mathrm{m}}$ values of $\mathrm{NAD}^{+}$for CD38 enzyme. ${ }^{33,34}$ These results suggest that by mimicking $\mathrm{NAD}^{+}, \mathrm{S}^{-\mathrm{NAD}^{+}}$could bind to the active site of human CD38 to inhibit its enzymatic activity and a single atomic substitution within the ribosyl ring seems to induce no adverse effects on its binding to the CD38 enzyme. Additionally, no slow-onset inhibition was observed for $\mathrm{S}^{-\mathrm{NAD}^{+}}$in direct competition assays with extended incubation or in studies of preincubating CD38 with S-NAD ${ }^{+}$.

In addition to human CD38, the chemical stability of S-NAD ${ }^{+}$ was evaluated for human sirtuin 2 (SIRT2), an NAD ${ }^{+}$-dependent protein deacetylase. Trypsin-coupled fluorescence-based activity assays indicated that in contrast to $\mathrm{NAD}^{+}$that could participate in SIRT2-catalyzed protein deacetylation, S-NAD ${ }^{+}$ alone revealed no substrate activity for SIRT2 after overnight incubation with SIRT2 and a fluorogenic, acetylated peptide substrate and co-incubation of S-NAD ${ }^{+}$with $\mathrm{NAD}^{+}$significantly inhibited protein deacetylation catalyzed by SIRT2 (Fig. S8 These results suggest that as a stable mimic of $\mathrm{NAD}^{+}, \mathrm{S}^{-\mathrm{NAD}^{+}}$ could bind to the SIRT2 active site and inhibit its catalytic activity. It is expected that the replacement of the endocyclic oxygen with less electronegative sulfur would reduce chemical reactivity of the $\mathrm{N}$-glycosidic bond, making it more resistant to enzymatic cleavage. Moreover, sulfur substitution may induce minimal changes in ribosyl geometry, causing little effects on enzyme binding. By altering bond stability and retaining

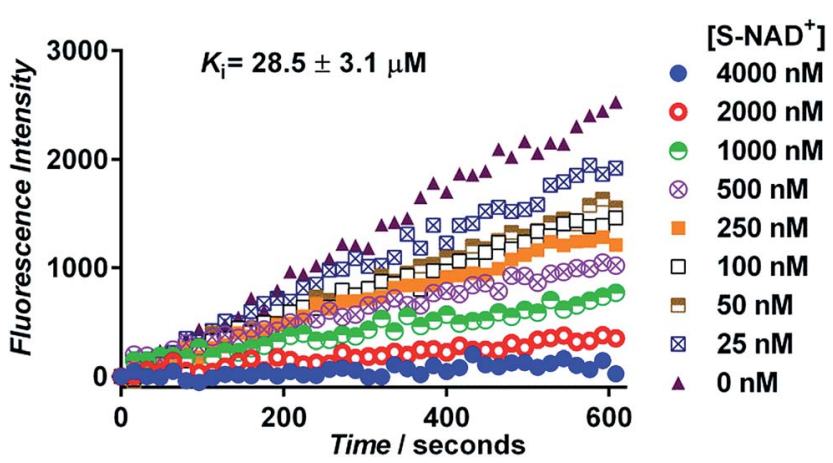

Fig. 4 Inhibition activity of S-NAD ${ }^{+}$for human $\mathrm{CD} 38$. Recombinant human CD38 ( $8 \mathrm{nM}$ ) was incubated with $50 \mu \mathrm{M} \mathrm{NGD}^{+}$in the presence of varied concentrations of S-NAD . CD38 cyclase activities were monitored on the basis of the formation of fluorescent cGDPR as measured at $410 \mathrm{~nm}$. 
molecular geometry, the single atomic substitution results in the $\mathrm{S}^{-\mathrm{NAD}^{+}}$as a stable mimic of $\mathrm{NAD}^{+}$.

In addition to enhancing stability of the $\mathrm{N}$-glycosidic linkage between nicotinamide and ribose ring, replacing the endocyclic oxygen with sulfur is expected to have no impact on reduction of nicotinamide ring in oxidation-reduction reactions. To determine whether S-NAD ${ }^{+}$could participate in electron transfer reactions, bovine glutamate dehydrogenase (GDH) and Leuconostoc mesenteroides glucose-6-phosphate dehydrogenase (G6PDH) were utilized, which are known to catalyze oxidation of glutamate and glucose-6-phosphate by $\mathrm{NAD}^{+}$, respectively. ${ }^{35,36}$ Enzymatic activity assays revealed that incubation of S-NAD ${ }^{+}$ with bovine GDH and L. mesenteroides G6PDH resulted in characteristic increases of UV absorbance at $340 \mathrm{~nm}$ in a manner similar to those of $\mathrm{NAD}^{+}$-containing reactions (Fig. S9:). HPLC analyses of the reduction of $\mathrm{NAD}^{+}$and S-NAD by bovine GDH and L. mesenteroides G6PDH showed enzymedependent formation of NADH and S-NADH peaks, which were confirmed by mass spectrometry (Fig. S10 and S11). These results suggest that the single atomic substitution within NR ribosyl ring has little effects on accepting electrons and $\mathrm{S}^{-\mathrm{NAD}^{+}}$can participate in redox reactions.

To explore the binding mode of S-NAD ${ }^{+}$with human CD38, $\mathrm{X}$-ray crystallographic analysis was carried out. The extracellular domain of wild-type human CD38 except four mutated glycosylation sites was recombinantly expressed in mammalian cells (Fig. S6:). Enzymatic activities of purified CD38 were verified by fluorescence- and HPLC-based activity assays using $\mathrm{NGD}^{+}$and $\mathrm{NAD}^{+}$as substrates. Recombinant human CD38 in high purity was successfully cocrystallized with S-NAD ${ }^{+}$and its X-ray crystal structure was solved at a resolution of $2.4 \AA$ (Fig. 5 and Table S2 $)$. The determined crystal structure clearly revealed the binding of S-NAD ${ }^{+}$to the active site of human $\mathrm{CD} 38$ and multiple interactions formed between $\mathrm{S}^{-\mathrm{NAD}^{+}}$and active site residues. The $4^{\prime}$-thioribose ring of $\mathrm{S}^{-\mathrm{NAD}^{+}}$adopted a $\mathrm{C}^{\prime}$-endo-C2 $2^{\prime}$-exo conformation. It was seen that the distances between S-NR $2^{\prime}-\mathrm{OH}$ of S-NAD ${ }^{+}$and side chains of S193 and E226 are $2.8 \AA$ and $3.6 \AA$, respectively. The S-NR $3^{\prime}-\mathrm{OH}$ of S-NAD ${ }^{+}$is $2.7 \AA$ away from the side chain of E146 (Fig. 5). These observations support that these three residues play important roles in catalysis of human CD38 and are consistent with previous reports, in which it was shown that E226 and S193 are catalytic residues involved in stabilization of formed oxacarbenium ion intermediate and E146 controls cyclizing and hydrolyzing activities. ${ }^{6,15,37}$ The most striking finding for the determined X-ray structure is that the nicotinamide ring of S-NAD ${ }^{+}$is fully stacked on top of the indole ring of W189 (Fig. 5). The planes of S-NAD ${ }^{+}$nicotinamide and W189 indole are nearly parallel with an interplanar spacing of approximately $3.4 \AA$ A. On the basis of the solved X-ray structure, the relative position, orientation, and distance of the nicotinamide moiety of S-NAD ${ }^{+}$at the active site of human $\mathrm{CD} 38$ suggest that W189 plays an important role in activation of the leaving group upon binding of substrate. This finding is consistent with previous studies which showed that mutations of W189 cause significantly decreased enzymatic activities of human CD38.

Comparative analysis of S-NAD ${ }^{+}$-bound $\mathrm{CD} 38$ with apo-CD38 (PDB ID: 1YH3) and NAD ${ }^{+}$-bound CD38 (E226Q) (PDB ID: 2I65)

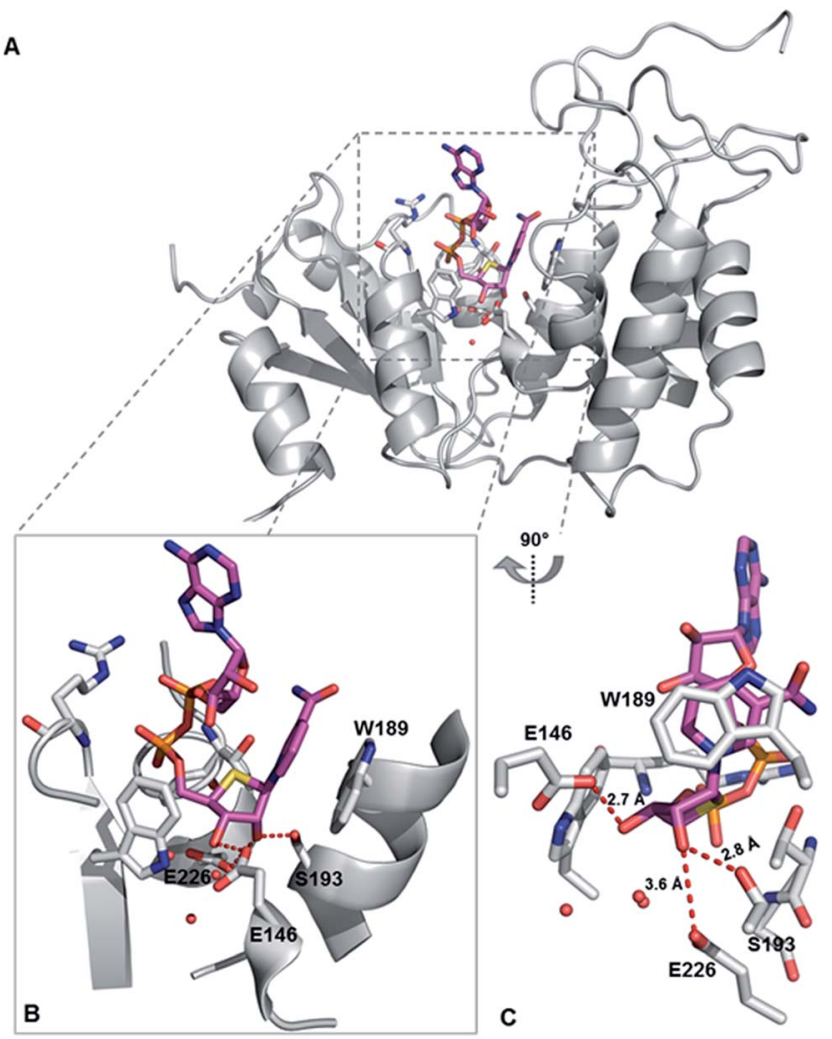

Fig. 5 X-ray crystal structure of catalytically active human CD38 in complex with S-NAD ${ }^{+}$. CD38 and $\mathrm{S}-\mathrm{NAD}^{+}$are shown in grey and magenta, respectively. (A) Overall crystal complex structure of human CD38-S-NAD ${ }^{+}$. (B) and (C) Bound S-NAD ${ }^{+}$at the active site of human CD38 with indicated catalytic residues and interacting water molecules. PDB ID: 6EDR.

showed no significant changes in overall structure (Fig. S12 ${ }^{15,38}$ Relative to S-NR of S-NAD ${ }^{+}$, the NR moiety of $\mathrm{NAD}^{+}$at the binding site of catalytically inactive CD38 (E226Q) is closer to the bottom of the binding pocket. As a result, the nicotinamide ring of $\mathrm{NAD}^{+}$forms very minor stacking interaction with the indole ring of W189 (Fig. S12 ). The nicotinamide rings of $\mathrm{NAD}^{+}$and S-NAD ${ }^{+}$at the $\mathrm{CD} 38$ active sites reveal distinct orientations with $\mathrm{C} 2-\mathrm{N} 1-\mathrm{C} 1^{\prime}-\mathrm{C} 2^{\prime}$ dihedral angles of $174.3^{\circ}$ and $34.7^{\circ}$, respectively, which enable the amide group of $\mathrm{NAD}^{+}$to be in hydrogen bond distance to the side chain of E146. In addition, the ribose ring of $\mathrm{NAD}^{+}$adopted a $\mathrm{C}^{\prime}$-endo-C3'-exo conformation. These structural differences between $\mathrm{NAD}^{+}$and $\mathrm{S}^{-\mathrm{NAD}^{+}}$at the active sites of $\mathrm{CD} 38$ suggest that the sulfur substitution results in an $\mathrm{NAD}^{+}$analogue more resistant to the $N$-glycosidic bond breakage. Taken together, the X-ray structure of S-NAD ${ }^{+}$with catalytically active human CD38 is consistent with the substrate and inhibition activities studies and demonstrates that as a stable mimic of $\mathrm{NAD}^{+}$, the generated S$\mathrm{NAD}^{+}$allows X-ray crystallographic characterization of reactive complexes of CD38 to elucidate its catalytic mechanism.

\section{Conclusions}

As a novel stable $\mathrm{NAD}^{+}$mimic, S-NAD ${ }^{+}$was successfully generated. By exploiting human NRK1 and NMNAT1, S-NAD ${ }^{+}$could 
be efficiently prepared from its chemically synthesized S-NR precursor through a two-step enzymatic process in a high yield. The generated S-NAD ${ }^{+}$is chemically inert to cleavage by human CD38 and sirtuin 2, while functioning as an electron acceptor in redox reactions. The X-ray structure of S-NAD ${ }^{+}$with human CD38 demonstrates its binding to enzyme active site and revealed residues important for catalysis along the reaction coordinate. This work provides a facile and efficient chemoenzymatic approach for the generation of $\mathrm{S}^{-\mathrm{NAD}^{+}}$and a unique and important tool that can be extended to investigate $\mathrm{NAD}^{+}$dependent enzymes.

\section{Conflicts of interest}

There are no conflicts of interest to declare.

\section{Acknowledgements}

We would like to thank the staff at Synchrotron Radiation Light Source (SSRL) (beamline 12-2) for their excellent support during data collections and Dr Gye Won Han for her advice on the refinement and checking the quality of the final structure. This work was supported by University of Southern California School of Pharmacy Start-Up Fund for New Faculty, Sharon L. Cockrell Cancer Research Fund, The $\mathrm{V}$ Foundation for Cancer Research V Scholar Grant V2016-021 (to Y. Z.), and University of Southern California Research Center for Liver Diseases Pilot Grant P30DK048522 (to Y. Z.).

\section{References}

1 K. W. Ryu, D. S. Kim and W. L. Kraus, New Facets in the Regulation of Gene Expression by ADP-Ribosylation and Poly(ADP-ribose) Polymerases, Chem. Rev., 2015, 115, 2453.

$2 \mathrm{H}$. Lin, Nicotinamide adenine dinucleotide: beyond a redox coenzyme, Org. Biomol. Chem., 2007, 5, 2541.

3 S. Imai and L. Guarente, NAD+ and sirtuins in aging and disease, Trends Cell Biol., 2014, 24, 464.

4 E. Verdin, $\mathrm{NAD}(+)$ in aging, metabolism, and neurodegeneration, Science, 2015, 350, 1208.

5 S. Yamamoto-Katayama, A. Sato, M. Ariyoshi, M. Suyama, K. Ishihara, T. Hirano, H. Nakamura, K. Morikawa and H. Jingami, Site-directed removal of N-glycosylation sites in BST-1/CD157: effects on molecular and functional heterogeneity, Biochem. J., 2001, 357, 385.

6 R. Graeff, C. Munshi, R. Aarhus, M. Johns and H. C. Lee, A single residue at the active site of CD38 determines its NAD cyclizing and hydrolyzing activities, J. Biol. Chem., 2001, 276, 12169.

7 E. Blacher, T. Dadali, A. Bespalko, V. J. Haupenthal, M. O. Grimm, T. Hartmann, F. E. Lund, R. Stein and A. Levy, Alzheimer's disease pathology is attenuated in a CD38-deficient mouse model, Ann. Neurol., 2015, 78, 88.

8 C. Escande, V. Nin, N. L. Price, V. Capellini, A. P. Gomes, M. T. Barbosa, L. O'Neil, T. A. White, D. A. Sinclair and E. N. Chini, Flavonoid apigenin is an inhibitor of the NAD+ ase CD38: implications for cellular NAD + metabolism, protein acetylation, and treatment of metabolic syndrome, Diabetes, 2013, 62, 1084.

9 M. G. Tarrago, C. C. S. Chini, K. S. Kanamori, G. M. Warner, A. Caride, G. C. de Oliveira, M. Rud, A. Samani, K. Z. Hein, R. Huang, D. Jurk, D. S. Cho, J. J. Boslett, J. D. Miller, J. L. Zweier, J. F. Passos, J. D. Doles, D. J. Becherer and E. N. Chini, A Potent and Specific CD38 Inhibitor Ameliorates Age-Related Metabolic Dysfunction by Reversing Tissue NAD(+) Decline, Cell Metab., 2018, 27, 1081. 10 J. Camacho-Pereira, M. G. Tarrago, C. C. S. Chini, V. Nin, C. Escande, G. M. Warner, A. S. Puranik, R. A. Schoon, J. M. Reid, A. Galina and E. N. Chini, CD38 Dictates AgeRelated NAD Decline and Mitochondrial Dysfunction through an SIRT3-Dependent Mechanism, Cell Metab., 2016, 23, 1127.

11 E. Blacher, B. Ben Baruch, A. Levy, N. Geva, K. D. Green, S. Garneau-Tsodikova, M. Fridman and R. Stein, Inhibition of glioma progression by a newly discovered CD38 inhibitor, Int. J. Cancer, 2015, 136, 1422.

12 F. Morandi, A. L. Horenstein, A. Chillemi, V. Quarona, S. Chiesa, A. Imperatori, S. Zanellato, L. Mortara, M. Gattorno, V. Pistoia and F. Malavasi, CD56brightCD16NK Cells Produce Adenosine through a CD38-Mediated Pathway and Act as Regulatory Cells Inhibiting Autologous CD4+ T Cell Proliferation, J. Immunol., 2015, 195, 965.

13 S. Chatterjee, A. Daenthanasanmak, P. Chakraborty, M. W. Wyatt, P. Dhar, S. P. Selvam, J. Fu, J. Zhang, H. Nguyen, I. Kang, K. Toth, M. Al-Homrani, M. Husain, G. Beeson, L. Ball, K. Helke, S. Husain, E. Garrett-Mayer, G. Hardiman, M. Mehrotra, M. I. Nishimura, C. C. Beeson, M. G. Bupp, J. Wu, B. Ogretmen, C. M. Paulos, J. Rathmell, X. Z. Yu and S. Mehrotra, CD38-NAD(+)Axis Regulates Immunotherapeutic Anti-Tumor $\mathrm{T}$ Cell Response, Cell Metab., 2018, 27, 85.

14 P. O. Hassa, S. S. Haenni, M. Elser and M. O. Hottiger, Nuclear ADP-ribosylation reactions in mammalian cells: where are we today and where are we going?, Microbiol. Mol. Biol. Rev., 2006, 70, 789.

15 Q. Liu, I. A. Kriksunov, R. Graeff, C. Munshi, H. C. Lee and Q. Hao, Structural basis for the mechanistic understanding of human CD38-controlled multiple catalysis, J. Biol. Chem., 2006, 281, 32861.

16 Q. Liu, I. A. Kriksunov, R. Graeff, H. C. Lee and Q. Hao, Structural basis for formation and hydrolysis of the calcium messenger cyclic ADP-ribose by human CD38, J. Biol. Chem., 2007, 282, 5853.

17 K. G. Hoff, J. L. Avalos, K. Sens and C. Wolberger, Insights into the sirtuin mechanism from ternary complexes containing NAD + and acetylated peptide, Structure, 2006, 14, 1231.

18 A. Zatorski, K. A. Watanabe, S. F. Carr, B. M. Goldstein and K. W. Pankiewicz, Chemical synthesis of benzamide adenine dinucleotide: inhibition of inosine monophosphate dehydrogenase (types I and II), J. Med. Chem., 1996, 39, 2422.

19 M. F. Langelier, L. Zandarashvili, P. M. Aguiar, B. E. Black and J. M. Pascal, NAD $(+)$ analog reveals PARP-1 substrate- 
blocking mechanism and allosteric communication from catalytic center to DNA-binding domains, Nat. Commun., 2018, 9, 844 .

20 B. G. Szczepankiewicz, H. Dai, K. J. Koppetsch, D. Qian, F. Jiang, C. Mao and R. B. Perni, Synthesis of carba-NAD and the structures of its ternary complexes with SIRT3 and SIRT5, J. Org. Chem., 2012, 77, 7319.

21 A. A. Sauve and V. L. Schramm, Mechanism-based inhibitors of CD38: a mammalian cyclic ADP-ribose synthetase, Biochemistry, 2002, 41, 8455.

22 J. H. Shrimp, J. Hu, M. Dong, B. S. Wang, R. MacDonald, H. Jiang, Q. Hao, A. Yen and H. Lin, Revealing CD38 cellular localization using a cell permeable, mechanismbased fluorescent small-molecule probe, J. Am. Chem. Soc., 2014, 136, 5656.

23 H. Jiang, J. Congleton, Q. Liu, P. Merchant, F. Malavasi, H. C. Lee, Q. Hao, A. Yen and H. Lin, Mechanism-based small molecule probes for labeling CD38 on live cells, J. Am. Chem. Soc., 2009, 131, 1658.

24 L. Sorci, F. Cimadamore, S. Scotti, R. Petrelli, L. Cappellacci, P. Franchetti, G. Orsomando and G. Magni, Initial-rate kinetics of human NMN-adenylyltransferases: substrate and metal ion specificity, inhibition by products and multisubstrate analogues, and isozyme contributions to NAD+ biosynthesis, Biochemistry, 2007, 46, 4912.

25 F. Berger, C. Lau, M. Dahlmann and M. Ziegler, Subcellular compartmentation and differential catalytic properties of the three human nicotinamide mononucleotide adenylyltransferase isoforms, J. Biol. Chem., 2005, 280, 36334.

26 W. Tempel, W. M. Rabeh, K. L. Bogan, P. Belenky, M. Wojcik, H. F. Seidle, L. Nedyalkova, T. Yang, A. A. Sauve, H. W. Park and C. Brenner, Nicotinamide riboside kinase structures reveal new pathways to NAD+, PLoS Biol., 2007, 5, e263.

27 L. S. Jeong, H. W. Lee, K. A. Jacobson, H. O. Kim, D. H. Shin, J. A. Lee, Z.-G. Gao, C. Lu, H. T. Duong and P. Gunaga, Structure - Activity Relationships of 2-Chloro-N 6substituted-4 '-thioadenosine-5 '-uronamides as Highly Potent and Selective Agonists at the Human A3 Adenosine Receptor, J. Med. Chem., 2006, 49, 273.

28 T. Yang, N. Y.-K. Chan and A. A. Sauve, Syntheses of nicotinamide riboside and derivatives: effective agents for increasing nicotinamide adenine dinucleotide concentrations in mammalian cells, J. Med. Chem., 2007, 50, 6458.

29 J. Dowden, C. Moreau, R. S. Brown, G. Berridge, A. Galione and B. V. Potter, Chemical synthesis of the second messenger nicotinic acid adenine dinucleotide phosphate by total synthesis of nicotinamide adenine dinucleotide phosphate, Angew. Chem., 2004, 116, 4737.

30 J. Dowden, R. S. Brown, C. Moreau, A. Galione and B. V. Potter, Chemical synthesis of the novel $\mathrm{Ca} 2+$ messenger NAADP, Nucleosides, Nucleotides Nucleic Acids, 2005, 24, 513.

31 Y. Cen and A. A. Sauve, Transition state of ADP-ribosylation of acetyllysine catalyzed by Archaeoglobus fulgidus Sir2 determined by kinetic isotope effects and computational approaches, J. Am. Chem. Soc., 2010, 132, 12286.

32 R. M. Graeff, T. F. Walseth, K. Fryxell, W. D. Branton and H. C. Lee, Enzymatic synthesis and characterizations of cyclic GDP-ribose. A procedure for distinguishing enzymes with ADP-ribosyl cyclase activity, J. Biol. Chem., 1994, 269, 30260.

33 G. Orsomando, V. Polzonetti and P. Natalini, NAD $(P)(+)-$ glycohydrolase from human spleen: a multicatalytic enzyme, Comp. Biochem. Physiol., Part B: Biochem. Mol. Biol., 2000, 126, 89.

34 K. A. Wall, M. Klis, J. Kornet, D. Coyle, J. C. Ame, M. K. Jacobson and J. T. Slama, Inhibition of the intrinsic NAD+ glycohydrolase activity of CD38 by carbocyclic NAD analogues, Biochem. J., 1998, 335(3), 631.

35 M. Li, C. J. Smith, M. T. Walker and T. J. Smith, Novel inhibitors complexed with glutamate dehydrogenase: allosteric regulation by control of protein dynamics, J. Biol. Chem., 2009, 284, 22988.

36 C. Olive, M. E. Geroch and H. R. Levy, Glucose 6-phosphate dehydrogenase from Leuconostoc mesenteroides. Kinetic studies, J. Biol. Chem., 1971, 246, 2047.

37 C. Munshi, R. Aarhus, R. Graeff, T. F. Walseth, D. Levitt and H. C. Lee, Identification of the enzymatic active site of CD38 by site-directed mutagenesis, J. Biol. Chem., 2000, 275, 21566.

38 Q. Liu, I. A. Kriksunov, R. Graeff, C. Munshi, H. C. Lee and Q. Hao, Crystal structure of human CD38 extracellular domain, Structure, 2005, 13, 1331. 